\title{
Pancreatitis aguda: una complicación infrecuente de las gastrostomías por migración del balón interno. Reporte de un caso y revisión de literatura
}

\section{Acute pancreatitis: A rare complication of gastrostomy due to intragastric balloon migration. Case report and literature review}

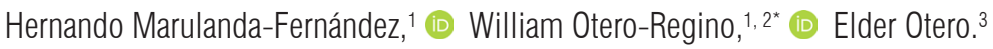

\author{
Gacceso abierto \\ Citación: \\ Marulanda-Fernández H, Otero-Regino W, \\ Otero E. Pancreatitis aguda: una complicación \\ infrecuente de las gastrostomías por \\ migración del balón interno. Reporte de un \\ caso y revisión de literatura. Rev Colomb \\ Gastroenterol. 2021;36(1):81-86. https://doi. \\ org/10.22516/25007440.427 \\ Gastroenterólogo, Universidad Nacional de \\ Colombia, Hospital Universitario Nacional de \\ Colombia. \\ 2 Profesor titular de Medicina, Unidad de \\ Gastroenterología, Universidad Nacional, \\ Hospital Universitario Nacional de Colombia. \\ ${ }^{3}$ Gastroenterólogo, Clínica Fundadores, Hospital \\ Central de la Policía. \\ *Correspondencia: William Otero Regino. \\ waoteror@gmail.com \\ Fecha recibido: $\quad 08 / 07 / 19$ \\ Fecha aceptado: 22/01/21

\section{(c) (1) $(9)$}

\begin{abstract}
Resumen
La pancreatitis aguda secundaria a la obstrucción de la papila mayor causada por la migración del balón interno es uno de los efectos secundarios poco frecuentes, pero potencialmente graves relacionados con el uso de gastrostomías endoscópicas percutáneas (PEG). Hasta ahora solo existen 15 casos reportados en el mundo, presentamos el que para nuestro conocimiento sería el caso número 16 en la literatura internacional.
\end{abstract}

Palabras clave

Pancreatitis, gastrostomía endoscópica percutánea (PEG), dolor abdominal, disfagia.

\section{Abstract}

Acute pancreatitis secondary to major papilla obstruction caused by intragastric balloon migration is one of the rare but potentially severe side effects associated with the use of percutaneous endoscopic gastrostomy (PEG). To date, there are only 15 cases reported worldwide. This article presents a case that, to the best of our knowledge, is the sixteenth case reported in the international literature.

\section{Keywords}

Pancreatitis, Percutaneous endoscopic gastrostomy (PEG), Abdominal pain, Dysphagia.

\section{INTRODUCCIÓN}

El uso de la gastrostomía endoscópica percutánea (PEG) como estrategia para garantizar el soporte nutricional ha incrementado de forma exponencial en las últimas dos décadas, en virtud de su sencillez y seguridad (1). El grupo de pacientes con indicaciones reales y que se benefician de este procedimiento es cada vez más selecto. Estudios recientes han cuestionado su práctica en pacientes con enfermedad cerebrovascular grave, mal pronóstico y demencia avanzada (2). A pesar de que no hay evidencia de que mejore la supervivencia, calidad de vida, o estado nutricional (3), continúa siendo implementada hasta en el $60 \%$ de los pacientes en las unidades de cuidado crónico (4), en el $10 \%$ de los pacientes de hogares geriátricos (5) y en el $5 \%$ de los pacientes mayores de 85 años (6). La tasa 
general de complicaciones varía entre el $1 \%$ y el $15 \%$ (7). Las complicaciones graves son poco frecuentes (8). Las complicaciones menores ocurren hasta en el $10 \%$ de los casos (9) y la mortalidad relacionada con el procedimiento es inferior al $1 \%$ (10). Entre las complicaciones mayores se encuentra la fascitis necrotizante; perforación intestinal, gástrica o esofágica; peritonitis; sangrado o desarrollo de fístulas (11). En el presente artículo se presenta el caso de un paciente con una complicación grave, poco frecuente y muchas veces no sospechada, la cual debe tenerse en cuenta dentro del diagnóstico diferencial de potenciales complicaciones relacionadas con la sonda de gastrostomía.

\section{CASO CLÍNICO}

La paciente es una mujer de 56 años con diagnóstico de disfagia de origen central, con gastrostomía desde hacía un año. Una semana después del cambio de gastrostomía por mal funcionamiento de la misma, consultó por dolor abdominal intenso, fiebre y vómito. Fue valorada por el servicio de cirugía general con sospecha de abdomen agudo. El cuadro hemático mostró leucocitosis con neutrofilia, perfil hepático con aumento de transaminasas ( 10 veces) y fosfatasa alcalina (3 veces). La tomografía abdominal con contraste mostró engrosamiento del bulbo y segunda porción del duodeno, vía biliar normal, sin cálculos en su interior, y cambios inflamatorios del cuerpo y la cola del páncreas sin compromiso de la grasa peripancreática. La conclusión del radiólogo fue "engrosamiento inespecífico del bulbo y la segunda porción duodenal" y "pancreatitis edematosa Baltazar A”. En los resultados de los exámenes tuvo amilasa sérica $432 \mathrm{U} / \mathrm{L}$ y lipasa $229 \mathrm{U} / \mathrm{L}$.

Como parte del estudio de pancreatitis aguda, en cirugía se solicitó una colangiorresonancia, y por el engrosamiento del duodeno en la tomografía axial computarizada (TAC), se solicitó endoscopia digestiva alta (EDA). En la endoscopia se encontró gastritis crónica y que el balón de la gastrostomía había migrado hacia el duodeno (Figura 1). En el hemicírculo posterior del bulbo, se encontró una úlcera de $10 \mathrm{~mm}$ cubierta por fibrina, que consideramos es secundaria al trauma ocasionado en ese sitio por la sonda. Al llegar a la segunda porción, se encontró que el balón de
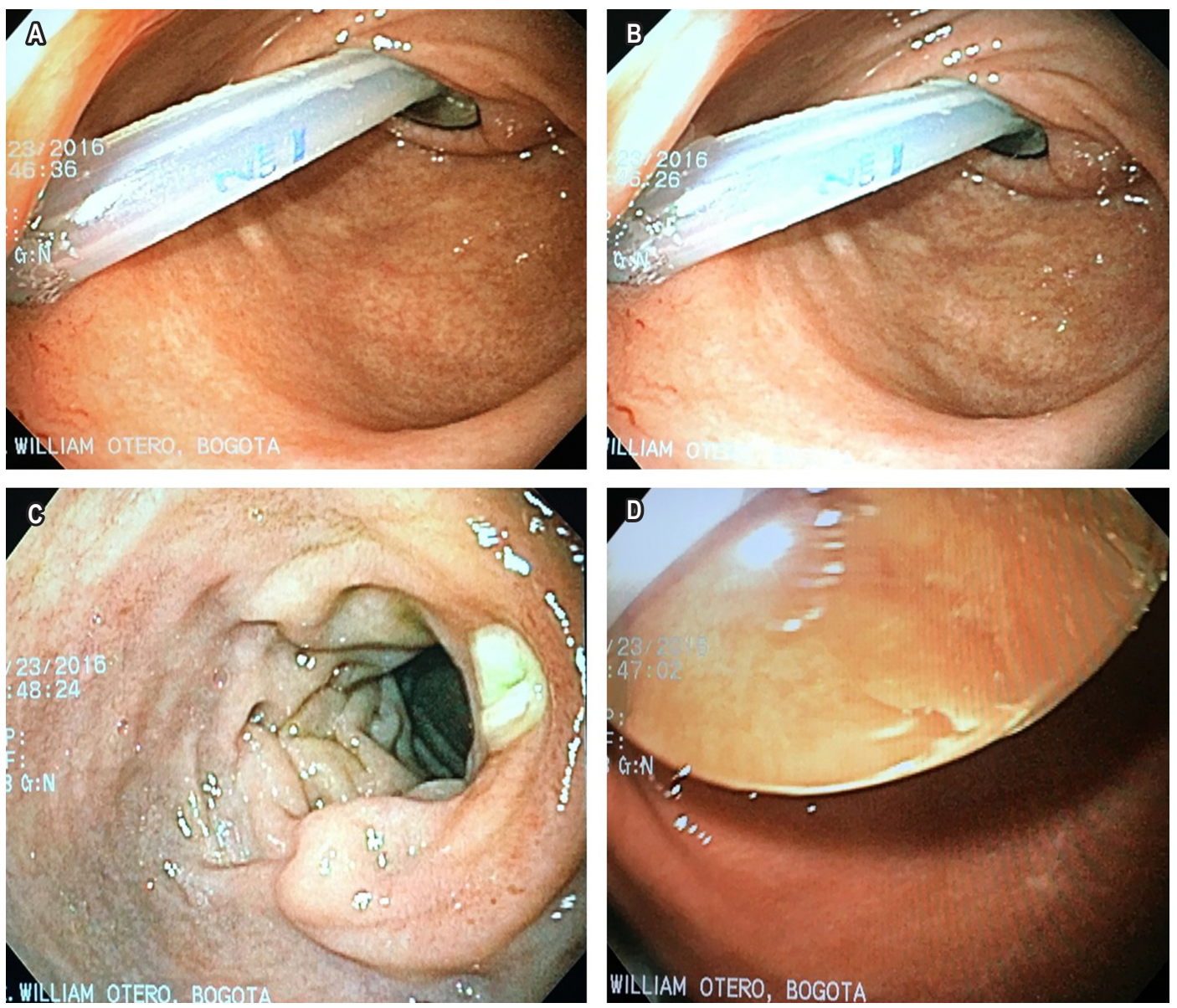

Figura 1. Balón de gastrostomía migrado a la segunda porción duodenal. 
la gastrostomía ocupaba el $80 \%$ de la luz. Inmediatamente se desinfló el balón, recuperando un volumen de $30 \mathrm{~mL}$, se traccionó la sonda y el balón se posicionó correctamente, y se llenó con solo $4 \mathrm{~mL}$ de solución salina, como indica el fabricante. Después de esta maniobra la paciente mejoró notablemente sus síntomas (quejido constante, movimientos con desasosiego y dolor).

Se modificó el tratamiento y se inició nutrición a través de la gastrostomía. El diagnóstico fue pancreatitis aguda secundaria a compresión de la papila mayor por el balón de gastrostomía y úlcera duodenal traumática por la sonda de gastrostomía. La elevación de las transaminasas se consideró secundaria a obstrucción biliar a nivel de la papila.

Se suspendió la orden de la colangiorresonancia. La paciente evolucionó favorablemente y nuevamente se educó a los cuidadores con respecto al manejo y cuidados de la sonda de gastrostomía, de acuerdo con nuestro protocolo de "educación a familiares y cuidadores" para el "manejo de sondas de gastrostomía". Al cuarto día se le dio salida sin complicaciones.

\section{DISCUSIÓN}

La PEG es en la actualidad el método de elección para alimentación enteral a largo plazo por tubos (12). Esta técnica fue descrita por primera vez en 1980 por Gauderer y colaboradores (13). Se considera un procedimiento seguro con pocas complicaciones (14). En la Tabla 1 se muestran las complicaciones potenciales relacionadas con el procedimiento y su frecuencia (15).

Tabla 1. Complicaciones relacionadas al uso de PEG

\begin{tabular}{|lc|}
\hline \multicolumn{1}{|c}{ Complicaciones } & Frecuencia \\
\hline Mayores & \\
\hline - Perforación intestinal & $1 \%-2 \%$ \\
\hline - Peritonitis & $1 \%$ \\
\hline - Broncoaspiración & $0,5 \%-1 \%$ \\
\hline - Sangrado & $1-5 \%$ \\
\hline - Fascitis necrotizante & $0,2 \%$ \\
\hline - Muerte & $0,5 \%-0,8 \%$ \\
\hline Menores & \\
\hline - Úlcera gástrica & $1 \%-2 \%$ \\
\hline - Infección periostomal & $5 \%-20 \%$ \\
\hline - Íleo & $1 \%-2 \%$ \\
\hline - Síndrome de buried bumper & $1 \%-3 \%$ \\
\hline
\end{tabular}

La pancreatitis aguda es una complicación poco frecuente, pero de las más graves, relacionada con el uso de gastrostomías y el primer caso fue descrito por Bui y colaboradores hace más de tres décadas (16). Esos autores plantearon la hipótesis de que la migración del balón generaba obstrucción y trauma mecánico directo sobre la papila mayor y menor, ocasionando edema e inflamación de la mucosa que impide el flujo a través del ducto pancreático (16). Desde entonces, se acepta que este es probablemente el mecanismo de la pancreatitis aguda. En una búsqueda de casos en Pubmed encontramos 14 casos publicados en la literatura mundial (Tabla 2).

Tabla 2. Casos descritos de pancreatitis aguda secundaria a migración del balón de gastrostomía en la literatura mundial

\begin{tabular}{|lcc|}
\hline \multicolumn{1}{|c}{ Estudio } & $\begin{array}{c}\text { Tipo de } \\
\text { sonda }\end{array}$ & $\begin{array}{c}\text { Tiempo de } \\
\text { inserción }\end{array}$ \\
\hline Bui y colaboradores, $1986(16)$ & Foley & 1 año \\
\hline Panicek y colaboradores, $1988(17)$ & Foley & ND \\
\hline Barthel y colaboradores, $1991(18)$ & Foley & 6 meses \\
\hline Duerksen y colaboradores, $2001(19)$ & PEG & 3 meses \\
\hline Miele y colaboradores, $2005(20)$ & Foley & Nd \\
\hline Imamura y colaboradores, $2007(21)$ & PEG & 1 mes \\
\hline Shah y colaboradores, $2012(22)$ & Foley & 24 horas \\
\hline Shah y colaboradores, $2012(22)$ & PEG & Nd \\
\hline Brauner E y colaboradores, $2014(15)$ & Foley & 1 año \\
\hline Taylor y colaboradores, $2016(23)$ & PEG & Nd \\
\hline Hawatmeh y colaboradores, 2016 (24) & PEG & 1 año \\
\hline Hawatmeh y colaboradores, 2016 (24) & PEG & 2 años \\
\hline Sekmenli y colaboradores, 2018 (25) & PEG & 2 meses \\
\hline Saleem S y colaboradores, 2018 (26) & PEG & 1 año \\
\hline Belat y colaboradores, 2019 (27) & PEG & 2 años \\
\hline
\end{tabular}

En los casos informados, esta complicación nunca ocurrió después de la colocación inicial del dispositivo. $\mathrm{Al}$ analizar cada uno de ellos, incluso en el nuestro, hubo un factor común desencadenante, que fue la manipulación reciente después del recambio de la sonda. La mayoría de los casos tuvo un desenlace favorable con una mejoría rápida de los síntomas y normalización de las alteraciones de laboratorio y de las imágenes. En la mitad de los casos, las sondas Foley fueron las responsables. Este tipo de sondas no están diseñadas para ser usadas como sondas de gastrostomía. La única indicación para utilizarlas es como medida transitoria para garan- 
tizar la permeabilidad del trayecto gastrocutáneo cuando se desaloja la sonda inicial, y a través de este, poder ingresar la sonda de gastrostomía de recambio. Adicionalmente, con la sonda Foley se presenta el inconveniente de la migración del balón, por no tener el tope externo para mantenerla fija en la piel (28). Por estas circunstancias, nuestro consejo es que esta sonda solo se utilice como medida temporal y no como una solución a largo plazo.

Cuando se necesita mantener la alimentación por sonda porque persiste la causa que ha motivado la inserción, debe utilizarse la sonda de recambio y sustituir la sonda provisional cuanto antes. La vida media del tubo de gastrostomía es de 12-15 meses, dependiendo de los cuidados que se tenga con la misma (29). Tenemos pacientes cuya sonda permanece funcionante hasta dos años. Las causas más frecuentes para el recambio de la sonda son perforación de esta, ruptura del balón, fugas o complicaciones infecciosas locales (30). En el estudio de Atencio D y colaboradores, se informó como causa de recambio el desplazamiento en el 12,5\%, deterioro de la sonda en 7,3 \% y ruptura del balón en 4,1 \% (31). Con frecuencia, los cuidadores manifiestan inquietud e inconformidad con respecto a cambios en el color, aspecto o deformidad del dispositivo externo y "exigen" el cambio de la sonda. Sin embargo, si esta funciona correctamente, no es necesario cambiarla ya que el cambio innecesario implica sobrecostos para el sistema de salud (32). En otras ocasiones y en nuestra experiencia, los familiares motu proprio suministran los alimentos muy calientes y esa temperatura produce deterioro con porosidad y, con el tiempo, mal olor de la sonda. En esas circunstancias, es necesario cambiarla y debe aprovecharse la ocasión para hacer la pedagogía correspondiente sobre los cuidados de la sonda.

En general, no hay evidencia de buena calidad sobre el beneficio de la verificación periódica de la ubicación (33) y solo se debe realizar cuando existan dudas sobre el adecuado funcionamiento, se verifique en una consulta específica si la sonda está funcionante y se evalúe la posibilidad de síndrome de buried bumper (34). En esa consulta, se observa la ubicación del botón externo, se introduce la sonda constatando que ingresa sin inconvenientes y, así mismo, se realiza la maniobra del giro libre, que consiste en verificar que, al girar la sonda, no haya dificultad para su rotación. De igual manera, se verifica que en ninguna parte exista microperforaciones. Finalmente, si la sonda tiene balón, este se desinfla y se verifica que no tenga más volumen del recomendado, se llena el balón con el volumen mínimo recomendado por el fabricante y se desplaza el tope externo sobre la marca deseada (35).

\section{CONCLUSIÓN}

La pancreatitis aguda es una complicación poco frecuente, pero potencialmente grave, con el uso de gastrostomías y su principal causa es secundaria a la obstrucción de la papila al migrar el balón hacia la segunda porción del duodeno. Esta complicación se da generalmente después de un recambio incorrecto de la sonda, en el que se llena de manera exagerada el balón y no se fijar externamente para evitar su desplazamiento. Existe una falsa creencia por parte de médicos y cuidadores, quienes consideran que al utilizar un mayor volumen para insuflar el balón hay un menor riesgo de que el paciente presente fugas a través de la ostomía o que logren retirarse de forma voluntaria o involuntaria la gastrostomía. Por el contrario, nuestra recomendación es que el balón se debe insuflar con el volumen mínimo necesario, según lo establecido por el fabricante, con el fin de evitar esta y otro tipo de complicaciones graves. Reiteramos, por ende, la necesidad de que su manipulación sea realizada exclusivamente por personas altamente capacitadas y entrenadas en su uso.

\section{Agradecimientos}

Agradecemos inmensamente a las doctoras de la Universidad Nacional de Colombia, Ana María González V (residente de endocrinología) y Johanna Buitrago Laguado (fellow de gastroenterología) por la lectura crítica del manuscrito y sus recomendaciones, que permitieron una mejor versión del mismo.

\section{REFERENCIAS}

1. John BK, Bullock M, Brenner L, McGaw C, Scolapio JS. Nutrition in the elderly. Frequently asked questions. Am J Gastroenterol. 2013;108(8):1252-66; quiz 1267. https:// doi.org/10.1038/ajg.2013.125

2. Mitchell SL, Teno JM, Kiely DK, Shaffer ML, Jones RN, Prigerson HG, Volicer L, Givens JL, Hamel MB. The clinical course of advanced dementia. N Engl J Med.
2009;361(16):1529-38. https://doi.org/10.1056/ NEJMoa0902234

3. Skelly RH. Are we using percutaneous endoscopic gastrostomy appropriately in the elderly? Curr Opin Clin Nutr Metab Care. 2002;5(1):35-42. https://doi. org/10.1097/00075197-200201000-00007 
4. Volkert D, Berner YN, Berry E, Cederholm T, Coti Bertrand P, Milne A, Palmblad J, Schneider S, Sobotka L, Stanga Z; DGEM (German Society for Nutritional Medicine), Lenzen-Grossimlinghaus R, Krys U, Pirlich M, Herbst B, Schütz T, Schröer W, Weinrebe W, Ockenga J, Lochs H; ESPEN (European Society for Parenteral and Enteral Nutrition). ESPEN Guidelines on Enteral Nutrition: Geriatrics. Clin Nutr. 2006;25(2):330-60. https://doi.org/10.1016/j.clnu.2006.01.012

5. Home enteral tube feeding in adults. En: Jones B, Holden C, Dalzell M, Micklewright A, Glencorse C. Annual BANS Report. Artificial Nutrition Support in the UK 2005. Reino Unido: BAPEN; 2005. p. 13-17.

6. Yriberry S, Monge V, Cabrera F, Barriga E, Vesco E. Gastrostomía endoscópica percutánea: experiencia prospectiva de un centro privado nacional. Rev Gastroenterol Peru. 2004;24(4):314-22.

7. Slater R. Percutaneous endoscopic gastrostomy feeding: indications and management. Br J Nurs. 2009;18(17):1036-43. https://doi.org/10.12968/ bjon.2009.18.17.44156

8. Razavi F, Gross S, Katz S. Endoscopy in the elderly: risks, benefits, and yield of common endoscopic procedures. Clin Geriatr Med. 2014;30(1):133-47. https://doi. org/10.1016/j.cger.2013.10.010

9. Belda O, Serrano P, Bozada JM, Fraile J, Garrido M, Guerrero R, Fenoy JL, García-Luna PP. La gastrostomía endoscópica percutánea. Realidad en la práctica nutricional clínica intra y extrahospitalaria. Rev Clin Esp. 2005;205(10):472-7. https://doi.org/10.1157/13079760

10. Sampson EL, Candy B, Jones L. Enteral tube feeding for older people with advanced dementia. Cochrane Database Syst Rev. 2009;2009(2):CD007209. https://doi. org/10.1002/14651858.CD007209.pub2

11. Chicharro L, Puiggrós C, Cots I, Pérez-Portabella C, Planas M. Complicaciones inmediatas de la gastrostomía percutánea de alimentación: 10 años de experiencia. Nutr Hosp. 2009;24(1):73-6.

12. Gauderer MW, Ponsky JL, Izant RJ Jr. Gastrostomy without laparotomy: a percutaneous endoscopic technique. J Pediatr Surg. 1980;15(6):872-5. https://doi.org/10.1016/ s0022-3468(80)80296-x

13. Wanden-Berghe C, Muñoz J, Cantó C, Domenech MD, Reyes MD, Pérez Moya C, Sanz Valero J. Gastrostomía Endoscópica Percutánea (PEG): diez años de experiencia. Nutr Hosp. 2010;25(6):949-53.

14. Bhat M, Bridges E. Acute obstructive pancreatitis caused by a migrated balloon gastrostomy tube. CMAJ. 2011;183(11):E759. https://doi.org/10.1503/ cmaj. 101198

15. Brauner E, Kluger Y. Gastrostomy tube dislodgment acute pancreatitis. World J Emerg Surg. 2014;9(1):23. https:// doi.org/10.1186/1749-7922-9-23

16. Bui HD, Dang CV. Acute pancreatitis: a complication of Foley catheter gastrostomy. J Natl Med Assoc. $1986 ; 78(8): 779-81$
17. Panicek DM, Ewing DK, Gottlieb RH, Chew FS. Gastrostomy tube pancreatitis. Pediatr Radiol. 1988;18(5):416-7. https://doi.org/10.1007/BF02388052

18. Barthel JS, Mangum D. Recurrent acute pancreatitis in pancreas divisum secondary to minor papilla obstruction from a gastrostomy feeding tube. Gastrointest Endosc. 1991;37(6):638-40. https://doi.org/10.1016/s00165107(91)70875-4

19. Duerksen DR. Acute pancreatitis caused by a prolapsing gastrostomy tube. Gastrointest Endosc. 2001;54(6):792-3. https://doi.org/10.1067/mge.2001.119600

20. Miele VJ, Nigam A. Obstructive jaundice and pancreatitis secondary to percutaneous endoscopic gastrostomy tube migration. J Gastroenterol Hepatol. 2005;20(11):1802-3. https://doi.org/10.1111/j.1440-1746.2005.04017.x

21. Imamura H, Konagaya $\mathrm{T}$, Hashimoto $\mathrm{T}$, Kasugai K. Acute pancreatitis and cholangitis: a complication caused by a migrated gastrostomy tube. World J Gastroenterol. 2007;13(39):5285-7. https://doi.org/10.3748/wjg.v13. i39.5285

22. Shah AM, Shah N, DePasquale JR. Replacement gastrostomy tube causing acute pancreatitis: case series with review of literature. JOP. 2012;13(1):54-7.

23. Taylor DF, Cho R, Cho A, Nguyen V, Sunnapwar A, Womeldorph C. Obstructive Acute Pancreatitis Secondary to PEG Tube Migration. ACG Case Rep J. 2016;3(4):e150. https://doi.org/10.14309/crj.2016.123

24. Hawatmeh A, Alkhateeb A, Arqoub AA, Jumean K, Shaaban $\mathrm{H}$. Gastrostomy tube migration complicated with acute pancreatitis: Two case reports with review of literature. Int J Crit Illn Inj Sci. 2016;6(1):48-50. https://doi. org/10.4103/2229-5151.177360

25. Sekmenli T, Gündüz M, Ciftci I, Emiroğlu HH, Koplay M. A Rare Cause of Acute Pancreatitis: Gastrostomy Catheter Migration. Arch Argent Pediatr. 2018;116(1):e183-e185.

26. Saleem S, Bleibel W. Stomach Ulcer as a Rare Cause of Pancreatitis: An Unusual Complication of a Displaced Percutaneous Endoscopic Gastrostomy Tube. Cureus. 2018;10(7):e2926. https://doi.org/10.7759/cureus.2926

27. Belat AM, Haquin A, O’Brien M, Goutelle S, de la Gastine $B$. Pancréatite aiguë en lien avec la migration d'une sonde de gastrostomie : à propos d'un cas et revue de la littérature. Presse Med. 2019;48(5):563-567. https://doi. org/10.1016/j.lpm.2019.04.007

28. Mic-Key, sonde d'alimentation par gastrostomie. Guide de bonnes pratiques de soins. Halyard Health; 2015.

29. Peñaloza A, Suárez J, Blanco L, Peñaloza A. Gastrostomía endoscópica percutánea: ¿Es éticamente aceptable? Rev Col Gastroenterol. 2013;28(2):150-60.

30. Arab K, Petit A. Complications des gastrostomies percutanées (hors complications immédiates). Nutr Clin Metab. 2011;25(3):190-5. https://doi.org/10.1016/j. nupar.2011.06.005

31. Atencio D; Blanco A, Otero W. Gastrostomía endoscópica percutánea en ancianos: indicaciones, seguridad y desenlaces. Rev Col Gastroenterol. 2015;30(1):3-10. 
32. Körner U, Bondolfi A, Bühler E, Macfie J, Meguid MM, Messing B, Oehmichen F, Valentini L, Allison SP. Ethical and legal aspects of enteral nutrition. Clin Nutr. 2006;25(2):196-202. https://doi.org/10.1016/j. clnu.2006.01.024

33. Moreno N, Otero W, Gómez M, Bula R, Otero E. Síndrome de "buried bumper" (botón interno de la gastrostomía enterrado): “desenterrando la solución”. Rev Col Gastroenterol. 2007;22(1):51-7.
34. Sebastian JJ. Gastrostomía endoscópica percutánea. Técnica e indicaciones. Endocrinol Nutr. 2004;51(4):15862. https://doi.org/10.1016/S1575-0922(04)74601-X

35. Erdogan A. Single endoscopist-performed percutaneous endoscopic gastrostomy tube placement. World J Gastroenterol. 2013;19(26):4172-6. https://doi. org/10.3748/wjg.v19.i26.4172 